\title{
Ansiedad estado y ansiedad rasgo en bailarines según el tipo de danza que practican y su condición como bailarín
}

María Liliana Valle Universidad de Lima

\author{
Recibido: 17 de mayo de 2014 / Aprobado: 16 de junio de 2014
}

Se determinó comparativamente la ansiedad estado y la ansiedad rasgo en bailarines según el tipo de danza escogido (clásica y contemporánea) y su condición como bailarín (profesional y amateur). Se trabajó con 58 bailarines de Lima Metropolitana, de los cuales el 24,1 \% fueron bailarines clásicos amateur; el 27,6\%, bailarines clásicos profesionales; el 20,7\%, bailarines contemporáneos amateur, y 27,6\%, bailarines contemporáneos profesionales. Se utilizó el Inventario de Ansiedad Rasgo-Estado (IDARE). Los hallazgos indicaron que sí existen diferencias estadísticas significativas en la ansiedad estado entre bailarines de danza clásica profesionales y bailarines de danza clásica amateur, así como entre bailarines de danza contemporánea profesional y danza contemporánea amateur. Los bailarines clásicos profesionales y los bailarines contemporáneos amateur son los que presentan mayor ansiedad estado.

ansiedad estado / ansiedad rasgo / danza clásica / danza contemporánea

\section{State and trait anxiety in dancers according to dance type and condition as dancer}

The aim of this study was to determine comparatively state and trait anxiety on dancers according to the type of chosen dance (classical and contemporary) and their conditions as dancers (professional and amateur) using the State-Trait Anxiety Inventory (STAI) The sample of study was composed of 58 dancers in metropolitan Lima: 24,1\% were amateur classical dancers, 27,6\% professional classical dancers; 20,7\% amateur contemporary dancers; and 27,6\% professional contemporary dancers. Results show that there are significant statistical differences between professional and amateur classical dancers, and between professional and amateur and contemporary dancers. Classical professional dancers and amateur contemporary dancers show higher state anxiety.

state anxiety / trait anxiety / classic dance / contemporary dance 
La danza es, en esencia, la combinación de dos campos amplios: el arte y el deporte. Se le podría considerar una disciplina artística, cuyo desarrollo demanda del bailarín no solo un trabajo físico, sino también mental (Paparizos, Tripp, Sullivan \& Rubenstein, 2005). Actualmente, los avances en el área de la psicología del deporte han demostrado la importancia del trabajo no solo en el aspecto físico, sino también psicológico, con la finalidad de mejorar el rendimiento del deportista. Siendo, entonces, la danza, una combinación de arte con ejercicio físico, el trabajar tanto el aspecto psicológico como el físico está adquiriendo cada vez más importancia.

Por otro lado, las emociones se definen como reacciones psíquicas de intensidad particular; estas actúan, a la vez, sobre la conducta motora y van acompañadas sobre todo de fenómenos orgánicos. Por ello es que se afirma que pueden influir de manera notoria en el grado de esfuerzo que se debe realizar $y$, por consiguiente, en el resultado de los rendimientos. Las emociones pueden estimular a las personas que practican alguna actividad física y movilizarlas para que apliquen el máximo de sus fuerzas o disminuya su potencial. $\mathrm{Si}$ fuera el último caso, se convertiría en una persona indiferente y sin energía (García, 1997).

Las emociones negativas surgen cuando en las apreciaciones del sujeto aparecen señales amenazadoras, conflictos o frustraciones; mientras más intensos sean estos elementos valorativos, más fuertes son regularmente las reacciones emocionales (García, 1997). La ansiedad, considerada como una emoción negativa, a larga repercute significativamente en el desempeño de cualquier deportista o persona dedicada a la actividad física, como es el caso de los bailarines de $b a$ llet y de danza contemporánea.

El ballet clásico, como técnica, es una disciplina artística compuesta por un conjunto de movimientos y posiciones estilizadas que, conectados al ritmo de la música, llegan a expresar argumentos, estados de ánimo y al propio cuerpo en movimiento. Los bailarines se ven sometidos a un trabajo básicamente físico, en el cual se busca perfeccionar la ejecución del movimiento. No obstante, esta exigencia física se encuentra muy involucrada con una demanda emocional por parte de los mismos. La danza contemporánea también tiene sus propios requerimientos: el entrenamiento es constante y se plantea que el bailarín desarrolle su capacidad de improvisación y se ajuste a un tipo de danza libre de formalismos y estructuraciones. En ambos tipos de danza, para que la ejecución del movimiento sea eficiente y limpia, el bailarín debe saber controlar sus emociones (Clabaugh \& Morling, 2004; Taylor \& Taylor, 2008).

Dadas estas necesidades en los aspectos físico y emocional, los bailarines podrían estar presentando ciertos niveles de ansiedad ante la incertidum- 
bre del éxito o fracaso de sus ejecuciones o ante el apremio mismo de su entrenamiento y profesión en sí. Esta ansiedad experimentada puede ser un rasgo del propio bailarín (ansiedad rasgo) o puede aparecer según la situación a la cual se enfrente (un examen, competencia, actuaciones, entre otros) (Taylor \& Taylor, 2008).

Sobre la base de lo expuesto, se plantea la siguiente interrogante: ¿existen diferencias en la ansiedad estado y la ansiedad rasgo en grupos de bailarines, según el tipo de danza que practican y su condición como bailarín?

La presente investigación tiene el objetivo de determinar comparativamente la ansiedad estado y la ansiedad rasgo en bailarines según el tipo de danza escogido (clásica o contemporánea) y su condición como bailarín (profesional o amateur).

A la fecha se han reportado pocos estudios relacionados con el tema abordado en la presente investigación; sin embargo, se los referirá brevemente por la relación que enriquece y complementa el presente estudio.

En un estudio realizado por Price y Pettijohn II (2006) en Estados Unidos, se evaluó la percepción de bailarinas de ballet con relación a sí mismas y a su propio cuerpo. Para la investigación, se contó con una muestra compuesta por 38 bailarinas de ballet, las cuales fueron separadas aleatoriamente en dos grupos. Ambos grupos ejecutarían una clase de ballet, pero utilizando diferente vestimenta. Un grupo usó leotardo negro y mallas rosadas (uniforme establecido por la escuela de ballet), mientras que al otro grupo se le dio la opción de escoger su vestimenta para la clase, la cual podía ser ropa holgada. Al día siguiente, las participantes completaron una clase de ballet con la vestimenta contraria a la que habían usado. Después de las clases de cada día, las participantes respondieron una encuesta acerca de la percepción de su propio cuerpo y el nivel de rendimiento durante la clase. Los resultados indicaron que cuando las participantes vistieron el leotardo negro y las mallas rosadas, sus puntuaciones en la percepción de su propio cuerpo eran significativamente bajas, en comparación con el puntaje obtenido en la condición de vestir ropa holgada. Estos resultados confirman la hipótesis de que bailar en ropa ajustada (leotardo y mallas), en comparación con la ropa holgada, origina que las bailarinas de ballet se sientan más negativas con relación a su cuerpo, a sí mismas y a su rendimiento.

En otro estudio realizado por Adam, Brassington, Steiner y Matheson (2004) en Alemania, se buscó evaluar la calidad de sueño, los estados de ánimo y la actuación limitada de bailarines de ballet profesionales debida a lesiones físicas, para así poder identificar factores potenciales que pudieran ser incluidos en futuras intervenciones, para prevenir y tratar lesiones en 
la danza. La muestra empleada estuvo conformada por 30 bailarinas y 24 bailarines a quienes se les aplicó cinco cuestionarios: el Cuestionario Demográfico, la Escala de Estrés Percibido de Cohen, la Escala de Evaluación de Soporte Social, el Cuestionario de Perfiles de Estados de Ánimo y el Índice de Calidad de Sueño de Pittsburgh. Los resultados reportaron que la ausencia de los bailarines en actuaciones y ensayos debido a una lesión estaba significativa y positivamente correlacionada con estrés, perturbaciones en el sueño, adormecimiento durante el día y estados de ánimo negativos (como tensión, depresión, ira, fatiga y confusión). Por el contrario, esta misma ausencia correlacionaba significativa y negativamente con el soporte social.

Por último, en un estudio realizado con bailarinas de ballet y de danza moderna, en la Universidad de Delaware, en Newark, por Clabaugh y Morling (2004), se investigó sobre la precisión de los estereotipos de bailarinas de ballet y danza moderna preprofesionales con respecto a los rasgos de personalidad de cada tipo de bailarina, y sus autorreportes acerca del tipo de danza que ellas mismas desarrollaban. Todo ello con la finalidad de evaluar la precisión de los estereotipos de grupo. La muestra estuvo conformada por 89 bailarines preprofesionales, de los cuales 41 eran de ballet y 48 de danza moderna. Asimismo, para fines comparativos, en el estudio se contó con la participación de un grupo de no bailarines (quienes, no obstante, se relacionaban con la danza como espectadores o por haber bailado un tiempo no mayor a 10 años) conformado por 56 personas. Los instrumentos utilizados fueron un Cuestionario Demográfico, la Escala de Autoestima de un solo ítem de Robins (2001), la Escala de Temor a la Evaluación Negativa de Leary (1983), la Escala de Estima Corporal de Franzoi y Shields (1984), la Escala de la Necesidad Personal de Estructura de Neuberg y Newsom (1993), y la Escala de Deseabilidad de Control de Burger y Cooper (1979) (citados por Clabaugh y Morling, 2004). Entre los resultados, los participantes estereotiparon en forma precisa que las bailarinas de ballet puntuaban más alto que las de danza moderna, en cuanto al temor a la evaluación negativa y a la necesidad personal de estructura; asimismo, acertaron en la igualdad de los grupos con respecto a la estima con relación al desempeño físico. Con respecto a las escalas de estima corporal y autoestima, los bailarines sobreestimaron la diferencia entre bailarines clásicos y modernos. Los grupos pensaron que los bailarines de ballet tenían una imagen corporal pobre. En este caso, los bailarines exageraron la discrepancia entre ambos grupos en una dirección estereotipada. También se encontró que tanto los modernos como los clásicos están insatisfechos con sus cuerpos. 


\section{LA ANSIEDAD}

La ansiedad es una reacción adaptativa de emergencia ante situaciones que representen o sean interpretadas como una amenaza o peligro para la integridad psicofísica del individuo (Vallejo \& Gastó, 2000).

Es importante mencionar que, según Sierra, Ortega y Zubeidat (2003), en la actualidad es la psicología de la personalidad la que brinda solidez al concepto de ansiedad, mediante la concepción de esta última en términos de ansiedad rasgo y ansiedad estado. Además, Spielberger (1972, citado por Edelmann, 1992) sugiere que es importante que una adecuada teoría de la ansiedad distinga entre la ansiedad como un estado transitorio y la ansiedad como un rasgo relativamente estable de la personalidad. La relevancia de considerar la distinción entre la ansiedad como un estado transitorio y como una disposición relativamente estable de la personalidad proviene de los estudios hechos por Cattell y Scheier (1961) y Spielberger (1966). Cualquier conceptualización de la ansiedad debe tener en cuenta las diferencias individuales en su propensión, como una forma de vulnerabilidad relacionada con la ansiedad. Las diferencias estarán impresas en la evaluación que la persona haga de sus reacciones corporales y en la interpretación que realice de los eventos de su ambiente externo (Edelmann, 1992).
En el campo del ejercicio físico, la ansiedad no debe ser considerada como un factor negativo, puesto que para lograr un nivel óptimo de rendimiento, se considera necesario cierto grado de ansiedad, de manera que el individuo pueda estar alerta a lo que ocurre a su alrededor, emplear adecuadamente sus recursos y facilitar la activación necesaria en beneficio de su rendimiento. Sin embargo, cuando la ansiedad no permite, en este caso, al deportista o al bailarín, rendir adecuadamente ni desplegar sus habilidades al máximo, se convierte en un importante obstáculo (Lizárraga, 1999).

\section{DANZA CLÁSICA (BALLET) Y DANZA CONTEMPORÁNEA}

Para Castañer (2000), la danza, en su sentido más amplio, obedece a diversas finalidades, por ello se pueden diferenciar diversos tipos de danza. En primer lugar, está la danza de carácter académico, que también es conocida como danza clásica o ballet. Este tipo de danza se basa en una configuración de movimientos técnicos y evoluciones coreográficas mediante la repetición constante, el perfeccionamiento de la postura y los gestos precisos. Todo ello debe seguirse con rigurosidad. En segundo lugar, está la danza de carácter escénico, la cual tiene como finalidad la consecución de la llamada "obra artística" apta para el espectáculo. En tercer lugar, la danza de carácter místi- 
co, la cual se sustenta en conceptos mágicos y/o religiosos que, generalmente, se relacionan con la esfera tanto individual como social de los bailarines. En cuarto lugar, la danza también puede tener un carácter creativo-educativo, en el que se busca desarrollar la expresión e interpretación libre tanto de los movimientos corporales como de su composición coreográfica. En quinto lugar, la danza con carácter terapéutico busca influir sobre diferentes problemas del individuo interpretándolos a través de la gestualidad. Por último, en sexto lugar, la danza con carácter lúdico tiene el objetivo de favorecer las necesidades de distracción y de recreación de las personas.

Según Gaxotte (citado en Courbebaisse, 1974), la danza clásica es la danza de escuela. Se enseña, y, como toda enseñanza, tiene sus tradiciones y sus reglas. De acuerdo con Salazar (1955), la danza clásica se desarrolla sobre la base de un mecanismo riguroso, el cual, a partir de principios muy elementales (como es el caso de las seis posiciones de los pies), va complicando metódicamente y poco a poco los movimientos. Cuando los pasos más complicados tienen lugar, es posible descomponerlos en cada uno de los movimientos o piezas que los conforman, hasta encontrar, a la inversa, todos los pasos que se fueron integrando a aquel. En este tipo de danza nada se improvisa; todo está regulado matemáticamente. Esto se explica dado que en esta danza cada movimiento se basa en cada uno de los valores musicales del compás. De este modo, pareciera que los bailarines, al momento de danzar, solfeasen la música. Así, cuando se ingresa a una academia o compañía, se puede oír cómo los bailarines cuentan los grupos de cifras que miden la correspondencia entre los valores del compás y el movimiento plástico de pies, manos y cuerpo.

En cambio, la danza contemporánea se caracteriza por la libertad en todos los aspectos, mediante la cual muchas de las tradiciones y reglas impuestas por la danza clásica eran dejadas de lado. Se busca que el bailarín desarrolle la improvisación y queda exonerado de los formalismos de la danza de academia (Salazar, 1955). Según Gaxotte (citado en Courbebaisse, 1974), la danza contemporánea es una creación muy somera y parece haber sido inventada para los bailarines que, no sabiendo bailar, disimulan su insuficiencia bajo este tipo de danza. El baile contemporáneo cae en la peor trivialidad negativa: bailarines con pies desnudos, cuando los clásicos llevan zapatillas; bailarinas con malla, cuando las clásicas llevan túnicas o tutús; pies hacia adentro, cuando lo clásico exige todo hacia fuera; brazos como asas de cesto o mantenidos rígidos detrás de la espalda, cuando lo clásico busca la belleza del gesto.

En la danza contemporánea hay también un dominio del cuerpo y el espacio; sin embargo, la expresión corpo- 
ral es menos limitada, la interpretación es más libre. Además, la coreografía es más libre en el movimiento, debido a que el cuerpo tiene mayor margen para expresar todo lo que quiere. En este tipo de danza también se ocupan líneas, cruces, saltos, cambios, giros, etc. Los movimientos son más amplios, los papeles principales se interpretan de distintas maneras, con diversos tipos de movimiento. El cuerpo se mueve al unísono, pero sin formar diseños específicos como en el ballet (Bárcena, 1994).

\section{HiPÓTESIS DE LA INVESTIGACIÓN}

\section{Hipótesis generales}

$\mathrm{H}_{\mathrm{G} 1}$ : Existen diferencias en la ansiedad estado en bailarines según el tipo de danza al que se dedican (clásica o contemporánea) y su condición como bailarín (amateur o profesional).

$\mathrm{H}_{\mathrm{G} 2:}$ Existen diferencias en la ansiedad rasgo en bailarines según el tipo de danza al que se dedican (clásica o contemporánea) y su condición como bailarín (amateur o profesional).

\section{Hipótesis específicas}

$\mathrm{H}_{1}$ : $\quad$ Existen diferencias significativas en la ansiedad estado entre bailarines de danza clásica y danza contemporánea.

$\mathrm{H}_{2}$ : Existen diferencias significativas en la ansiedad rasgo entre baila- rines de danza clásica y danza contemporánea.

$\mathrm{H}_{3}$ : Existen diferencias significativas en la ansiedad estado entre bailarines amateur y profesionales.

$\mathrm{H}_{4}$ : $\quad$ Existen diferencias significativas en la ansiedad rasgo entre bailarines amateur y profesionales.

$\mathrm{H}_{5}$ : Existen diferencias significativas en la ansiedad estado entre bailarines de danza clásica profesionales y bailarines de danza clásica amateur, así como entre bailarines de danza contemporánea amateur y danza contemporánea profesionales.

$\mathrm{H}_{6}$ : Existen diferencias significativas en la ansiedad rasgo entre bailarines de danza clásica profesionales y bailarines de danza clásica amateur, así como entre bailarines de danza contemporánea amateur y danza contemporánea profesionales.

\section{Método}

\section{Diseño de investigación}

El diseño de la investigación es descriptivo comparativo (Hernández, Fernández \& Baptista, 2010), puesto que se recolectó información acerca de la ansiedad estado y la ansiedad rasgo en bailarines de danza clásica profesionales y amateur, y también en bailarines de danza contemporánea profesionales y amateur. Esto se realizó con el pro- 
pósito de comparar tanto la ansiedad estado como la ansiedad rasgo entre cada uno de los grupos.

\section{Participantes}

La muestra de participantes estuvo conformada por 58 bailarines entre hombres y mujeres, divididos en cuatro grupos, los cuales surgieron de la interacción de dos condiciones: el tipo de danza desarrollado y la condición del bailarín. El primer grupo estuvo constituido por 14 bailarines clásicos amateur; el segundo grupo por 16 bailarines clásicos profesionales; el tercer grupo por 12 bailarines contemporáneos amateur y el cuarto grupo por 16 bailarines contemporáneos profesionales (ver tabla 1).

Debido a las características del estudio, la muestra de participantes fue obtenida por medio de un muestreo no probabilístico de tipo intencional. Se contó con la participación de tres academias en las que se imparten clases de danza, tanto clásica como contem- poránea. También se tuvo el apoyo de un taller de una universidad particular, en la cual se imparten clases de danza contemporánea. Finalmente, en el estudio también participaron bailarines de dos compañías. Una de ellas, dedicada a la danza contemporánea únicamente, y la otra, centrada en la danza clásica. Es importante mencionar que todas estas academias, talleres y compañías son de Lima Metropolitana.

\section{Variables de estudio}

De acuerdo con lo planteado por Kerlinger y Lee (2001), la variable independiente asignada hace referencia a las características de la muestra. Se considera variable independiente asignada y variable dependiente sin que se trate necesariamente de un estudio causal. Por ello, en el presente estudio, las variables independientes asignadas son: "tipo de baile" (danza clásica y danza contemporánea) y "condición del bailarín" (profesional y amateur). Las variables depen-

Tabla 1

Grupos de bailarines según tipo de danza desarrollado y condición como bailarín

\begin{tabular}{lcc}
\hline Grupos & Frecuencia & Porcentaje \\
\hline Clásico amateur & 14 & 24,1 \\
Clásico profesional & 16 & 27,6 \\
Contemporáneo amateur & 12 & 20,7 \\
Contemporáneo profesional & 16 & 27,6 \\
\hline Total & 58 & 100,0 \\
\hline
\end{tabular}


dientes son: "ansiedad rasgo" y "ansiedad estado". Finalmente, las variables controladas son: "tiempo dedicado a la danza" (por lo menos un año) y "edad de los bailarines" (por lo menos 12 años).

\section{Instrumentos de recolección de datos}

Se utilizó el Inventario de Ansiedad Rasgo-Estado (IDARE), que está compuesto por 40 ítems, los cuales se distribuyen en dos escalas: 20 en la escala que mide la ansiedad estado y los otros 20 en la escala que mide la ansiedad rasgo.

Las puntuaciones que se obtienen en el IDARE varían desde una puntuación mínima de 20 hasta una puntuación máxima de 80 , tanto para la escala de ansiedad estado como para la escala de ansiedad rasgo. De esta manera, mientras más se acerque el puntaje a 80 , el nivel de ansiedad será más alto. Los evaluados responden a cada uno de los ítems valorándose ellos mismos en una escala de cuatro puntos. En el caso de la escala A-Estado, las cuatro categorías son: 1. No en lo absoluto. 2. Un poco. 3. Bastante. 4. Mucho. En la escala A-Rasgo, las categorías son: 1. Casi nunca. 2. Algunas veces. 3. Frecuentemente. 4. Casi siempre (Spielberger \& Díaz-Guerrero, 1970).

Algunos de los reactivos del IDA$\mathrm{RE}$ se formularon de forma tal que una valoración de 4 indica un alto nivel de ansiedad. Mientras que otros reactivos se formularon de forma inversa, es decir, una valoración alta indica muy poca ansiedad. La escala A-Estado está compuesta por 10 ítems con calificación directa y 10 ítems con calificación invertida. En cambio, la escala A- Rasgo tiene 7 reactivos invertidos y 13 reactivos de calificación directa. Es importante mencionar que los ítems invertidos en la escala A-Estado son el 1, 2, 5, 8, $10,11,15,16,19$ y 20 . En el caso de la escala A-Rasgo, los reactivos invertidos son $21,26,27,30,33,36$ y 39 (Spielberger \& Díaz-Guerrero, 1970).

\section{Análisis psicométrico del IDARE}

En la presente investigación, el análisis psicométrico de los resultados del IDARE se ejecutó por escalas. Tanto en la escala A-Estado (SXE) como en la escala A-Rasgo (SXR), cada una constituida por 20 ítems, el análisis psicométrico evidenció que la confiabilidad por consistencia interna de las dos escalas, evaluadas con el coeficiente alfa de Cronbach, alcanza valores que, según los criterios establecidos por Nunnally y Bernstein (1995), pueden estimarse como adecuados (ver tabla 2).

En el presente estudio, debido a que las normas del IDARE corresponden a una realidad diferente de la peruana $\mathrm{y}$, además, dada la antigüedad de los baremos trabajados en el instrumento, se decidió calificar teniendo en cuenta, para el análisis estadístico, el puntaje bruto o directo obtenido con base en los criterios de calificación propuestos por el mismo Spielberger (1970), los cua- 
Tabla 2

Análisis de ítems y de la confiabilidad del IDARE: ansiedad estado y ansiedad rasgo

\begin{tabular}{|c|c|c|c|c|c|c|c|c|c|}
\hline \multicolumn{5}{|c|}{ ANSIEDAD ESTADO } & \multicolumn{5}{|c|}{ ANSIEDAD RASGO } \\
\hline ÍTEM & Media & $\begin{array}{c}\text { Des- } \\
\text { viación } \\
\text { típica }\end{array}$ & $\begin{array}{l}\text { Corre- } \\
\text { lación } \\
\text { elemento- } \\
\text { total } \\
\text { corregida }\end{array}$ & $\begin{array}{c}\text { Alfa de } \\
\text { Cronbach } \\
\text { si se } \\
\text { elimina el } \\
\text { elemento }\end{array}$ & ÍTEM & Media & $\begin{array}{l}\text { Des- } \\
\text { viación } \\
\text { típica }\end{array}$ & $\begin{array}{l}\text { Correlación } \\
\text { elemento- } \\
\text { total } \\
\text { corregida }\end{array}$ & $\begin{array}{c}\text { Alfa de } \\
\text { Cronbach } \\
\text { si se } \\
\text { elimina el } \\
\text { elemento }\end{array}$ \\
\hline 1 & 2,07 & ,814 &, 564 & ,876 & 21 & 1,66 & ,690 & ,339 & ,809 \\
\hline 2 & 2,02 & ,761 &, 503 & ,878 & 22 & 1,76 &, 572 & ,094 & ,818 \\
\hline 3 & 1,84 & ,670 & ,493 & ,878 & 23 & 1,64 & ,693 & ,303 & ,811 \\
\hline 4 & 1,36 & ,552 & ,669 & ,874 & 24 & 1,57 & ,752 & ,335 & ,809 \\
\hline 5 & 1,83 & ,775 & ,612 & ,874 & 25 & 1,83 & ,625 & ,288 & ,811 \\
\hline 6 & 1,31 &, 537 & ,394 & ,881 & 26 & 2,62 & ,970 &, 551 & ,796 \\
\hline 7 & 1,81 & ,826 & ,422 & ,881 & 27 & 2,41 & ,937 & ,460 & ,802 \\
\hline 8 & 2,83 & ,901 & ,520 & ,877 & 28 & 1,47 & ,627 & ,599 & ,799 \\
\hline 9 & 1,71 & ,726 & ,472 & 879 & 29 & 2,00 & ,858 & ,595 & ,795 \\
\hline 10 & 1,97 & ,620 & ,547 & ,877 & 30 & 1,60 & ,748 & ,454 & 803 \\
\hline 11 & 1,84 & ,721 & ,375 & ,882 & 31 & 2,26 & 890 & ,427 & 804 \\
\hline 12 & 1,45 & ,626 & ,498 & 878 & 32 & 1,72 & 812 & ,591 & 796 \\
\hline 13 & 1,59 & ,750 & ,323 & ,883 & 33 & 1,91 & 801 & ,546 & ,798 \\
\hline 14 & 1,17 & ,534 & ,484 & ,879 & 34 & 2,64 & 1,119 & ,065 & 831 \\
\hline 15 & 2,60 & ,917 & ,626 & ,873 & 35 & 1,71 & ,817 & ,194 & ,817 \\
\hline 16 & 1,95 & ,782 & ,520 & ,877 & 36 & 1,90 & ,718 & ,366 & 808 \\
\hline 17 & 1,76 & ,802 & ,585 & ,875 & 37 & 2,07 & 876 & ,342 & ,809 \\
\hline 18 & 1,33 & 632 & ,205 & 886 & 38 & 1,90 & ,949 & ,332 & 810 \\
\hline 19 & 1,88 & ,818 & ,502 & ,878 & 39 & 1,83 & ,798 & ,383 & ,807 \\
\hline 20 & 1,64 & ,693 & ,595 & ,875 & 40 & 2,02 & 868 & ,515 & ,799 \\
\hline \multicolumn{5}{|c|}{ Coeficiente alfa de Cronbach $=0,88$} & \multicolumn{5}{|c|}{ Coeficiente alfa de Cronbach $=0,82$} \\
\hline
\end{tabular}

les ya fueron desarrollados en párrafos precedentes.

Además, es importante mencionar que para el presente estudio se construyó una Encuesta Sociodemográfica, la cual tuvo como finalidad recabar información que permitiera enriquecer los resultados obtenidos por las escalas, así como profundizar con mayor rigurosidad el análisis de las mismas. 
Este instrumento estuvo conformado por 21 preguntas, de las cuales las 4 primeras están relacionadas con los datos de filiación de la participante; el contenido de las otras 17 se centra principalmente en aspectos relacionados con la danza, como la dedicación, tipo de baile, años de experiencia, estresores, entre otros.

\section{Procedimiento de ReColección de DATOS}

Con la finalidad de recolectar los datos para la investigación, se visitó tres academias en las que se imparten clases de danza, tanto clásica como contemporánea. Asimismo, se contó con el apoyo de un taller de una universidad particular, en la cual se imparten clases de danza contemporánea. Finalmente, en el estudio también participaron bailarines de dos compañías, una de danza clásica y otra de danza contemporánea.

La asistencia a dichas instituciones se llevó a cabo en diferentes días y horarios, para poder acceder a todos los grados o niveles considerados dentro del estudio (profesional y amateur).

En los ambientes asignados por las escuelas y academias para la aplicación del instrumento, se procedió a repartirlos a los participantes, siendo la Encuesta Socio-demográfica la que se encontraba en primer lugar, y luego el Inventario de Ansiedad Rasgo-Estado. Seguidamente, se die- ron las indicaciones necesarias y se respondió a las dudas presentadas por los participantes.

\section{Resultados}

\section{Instrumentos}

El IDARE como instrumento de medición de la ansiedad rasgo y la ansiedad estado presenta confiabilidad por consistencia interna a través del coeficiente alfa de Cronbach (ver tabla 2).

Los datos recabados con la Encuesta Sociodemográfica no ofrecen valores altamente significativos en cuanto a la diferenciación de la ansiedad estado y la ansiedad rasgo entre los grupos de bailarines. Por lo tanto, son utilizados únicamente para fines descriptivos de la muestra participante.

\section{Contraste de hipótesis}

Los resultados incluidos en la tabla 3 indican que en la comparación de la ansiedad Estado según el tipo de danza que practican los participantes, se encuentra que la prueba $t$ para grupos independientes alcanza un valor de $t=$ $0.15 p>.05$, el cual no es significativo. Por lo tanto, se concluye que las medias son similares, es decir, las diferencias que se encuentran son aleatorias, por lo cual la hipótesis específica 1 no es válida. Igualmente, en la comparación de la ansiedad rasgo según el tipo de danza desarrollado por los participantes, se aprecia que la prueba $t$ para grupos 
Tabla 3

Comparación de la ansiedad estado y la ansiedad rasgo, según el tipo de danza practicado

\begin{tabular}{|c|c|c|c|c|c|c|}
\hline & \multicolumn{2}{|c|}{$\begin{array}{l}\text { Danza clásica } \\
\qquad N=30\end{array}$} & \multicolumn{2}{|c|}{$\begin{array}{l}\text { Danza contemporánea } \\
\qquad N=28\end{array}$} & \multirow[b]{2}{*}{.$t$} & \multirow[b]{2}{*}{$p$} \\
\hline & $M$ & D. E. & $M$ & D. E. & & \\
\hline Ansiedad estado & 36,10 & 7,30 & 35,79 & 9,11 & 0.15 & .886 \\
\hline Ansiedad rasgo & 39,00 & 8,05 & 37,96 & 7,38 & 0.51 & .612 \\
\hline
\end{tabular}

independientes alcanza un valor de $t=$ $0.51 p>.05$, que no es significativo. Por eso, se afirma que las medias aritméticas son similares, es decir, las diferencias encontradas son aleatorias. Por ello se concluye que la hipótesis específica 2 no es válida.

Respecto de la comparación de la ansiedad estado según la condición del bailarín (ver tabla 4), utilizando la prueba $t$ para muestras independientes, se evidencia que el valor de $t=0.99 p$ $>.05$ no es significativo, con lo cual se puede concluir que las medias son similares, por lo que las diferencias halladas son aleatorias. Por lo tanto, la hipótesis específica 3 resulta no válida. Asimismo, los resultados evidencian que, en la comparación de la ansiedad rasgo según la condición de los bailarines, la prueba $t$ para grupos independientes alcanza un valor de $t=$ $0.91 p>.05$, el cual no es significativo. Con base en ello, se puede concluir que las medias son similares, es decir, las diferencias encontradas son aleatorias. Por tanto, la hipótesis específica 4 no es válida.

Tabla 4

Comparación de la ansiedad rasgo según la condición del bailarín

\begin{tabular}{lcccccc}
\hline & \multicolumn{3}{c}{$\begin{array}{c}\text { Amateur } \\
\mathbf{N}=\mathbf{3 6}\end{array}$} & \multicolumn{2}{c}{$\mathbf{N = \mathbf { 2 2 }}$} \\
& $\mathbf{M}$ & D. E. & $\mathbf{M}$ & D. E. & .t & p \\
\hline Ansiedad estado & 36,78 & 8,97 & 34,59 & 6,59 & 0.99 & .326 \\
Ansiedad rasgo & 39,22 & 7,94 & 37,32 & 7,27 & 0.91 & .364 \\
\hline
\end{tabular}


Tabla 5

Análisis de las medias de la ansiedad estado según el tipo de danza que desarrollan y la condición del bailarín

\begin{tabular}{lccc}
\hline $\begin{array}{l}\text { Tipo de danza que } \\
\text { desarrolla }\end{array}$ & $\begin{array}{c}\text { Lugar de la danza en su } \\
\text { vida }\end{array}$ & Media & Error típ. \\
\hline \multirow{2}{*}{ Clásica } & Profesión & 39,11 & 1,84 \\
& Amateur & 31,58 & 2,25 \\
Contemporánea & Profesión & 34,44 & 1,84 \\
& Amateur & 38,20 & 2,47 \\
\hline
\end{tabular}

Combinando los cuatro grupos de participantes, se puede apreciar que en el caso de la danza clásica, la media general de los bailarines profesionales con respecto a la ansiedad estado es de 39,11 (D. E. = 1,84); en cambio, la de los bailarines amateur es de 31,58 (D. E. $=2,25)$. En el caso de los bailarines contemporáneos, se observa que la media general es de 34,44 (D. E. $=1,84)$ en los profesionales y 38,20 (D. E. $=2,47)$ en los amateur. Es importante observar que en el caso de los bailarines clásicos, existen diferencias significativas entre las medias de los bailarines profesionales $(M=39,11, \mathrm{D}$. E. $=1,84)$ y amateur $(\mathrm{M}=31,58, \mathrm{D} . \mathrm{E}$. $=2,25$ ). En cuanto a los bailarines contemporáneos, se puede observar también diferencias en las medias de los bailarines profesionales $(M=34,44, \mathrm{D}$. E. $=1,84)$ y amateur o recreativos $(M$ $=38,20$, D. E. $=2,47$ ).

El gráfico de perfil de la escala de ansiedad estado (figura 1), muestra los niveles de dicha ansiedad en los participantes según el tipo de danza que desarrollan y su condición como bailarín. Se puede observar que en el grupo de los bailarines de danza clásica los profesionales denotan una mayor ansiedad estado en comparación con los amateur. En cambio, en el caso de los bailarines contemporáneos, son los amateur los que evidencian mayor ansiedad estado en comparación con los profesionales. Por lo tanto, la hipótesis específica 5 es válida.

Con respecto al análisis de medias de los grupos de participantes según el tipo de danza desarrollado y su condición como bailarín, se encuentra que entre los bailarines clásicos profesionales $(M=40,28$, D. E. $=1,84)$ y los bailarines clásicos amateur $(M=37,08$, D. E. = 2,25) la ansiedad rasgo se presenta con diferencias que no son considerablemente significativas. En referencia a los bailarines contemporáneos, se observa que la diferencia en 
Figura 1

Análisis de la interacción de la ansiedad estado entre el tipo de danza practicada y la condición del bailarín

\section{Medias marginales estimadas de A-ESTADO}

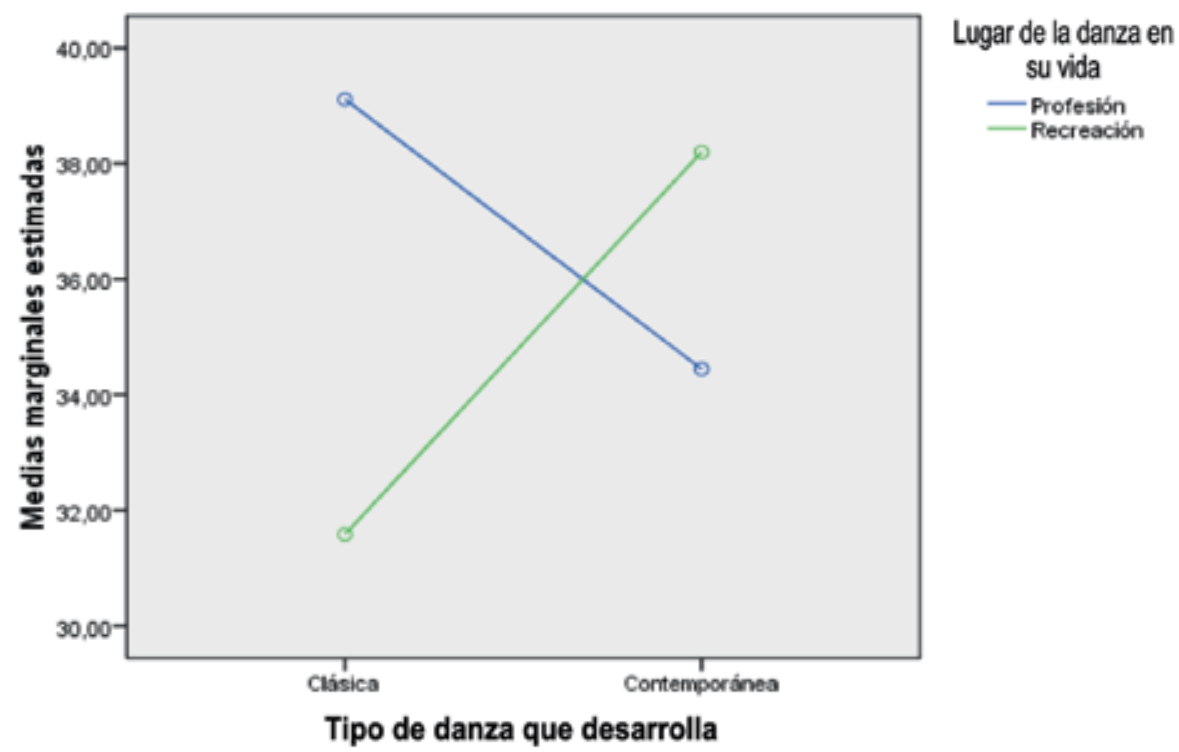

la ansiedad rasgo experimentada por los profesionales $(M=38,17$, D. E. $=1,84)$ y los amateur $(M=37,60$, D. E. $=2,46)$ no es significativa. Es importante también tomar en cuenta la diferencia en la ansiedad rasgo entre los bailarines profesionales clásicos $(M=40,28, \mathrm{D}$. E. $=1,84)$ y los profesionales contemporáneos $(M=38,17$, D. E. $=1,84)$, que, como puede apreciarse, esta existe pero no es tan significativa. En el caso de los bailarines amateur clásicos $(M=$ $37,08$, D. E. $=2,25)$ y los amateur contemporáneos $(M=37,60$, D. E. $=2,46)$, se observa que no existen diferencias significativas en cuanto a su vivencia de la ansiedad rasgo.

Según lo expuesto en el gráfico de perfil (figura 2), se puede observar que la ansiedad rasgo se experimenta en mayor medida en los bailarines profesionales clásicos, en comparación con los amateur clásicos; sin embargo, esta diferencia no es significativa. En el caso de los contemporáneos, se aprecia que los profesionales evidencian mayor ansiedad rasgo que los amateur; no obstante, esta diferencia no es significativa. Por consiguiente, la hipótesis específica 6 no es válida. 
Tabla 6

Análisis de las medias de la ansiedad rasgo según el tipo de danza que desarrollan y la condición del bailarín

\begin{tabular}{lccc}
\hline $\begin{array}{l}\text { Tipo de danza que } \\
\text { desarrolla }\end{array}$ & $\begin{array}{c}\text { Lugar de la danza en su } \\
\text { vida }\end{array}$ & Media & Error típ. \\
\hline \multirow{2}{*}{ Clásica } & Profesión & 40,28 & 1,84 \\
& Amateur & 37,08 & 2,25 \\
Contemporánea & Profesión & 38,17 & 1,84 \\
& Amateur & 37,60 & 2,46 \\
\hline
\end{tabular}

Figura 2

Análisis de la interacción de la ansiedad rasgo entre el tipo de danza practicada y la condición del bailarín

Medias marginales estimadas de A-RASGO

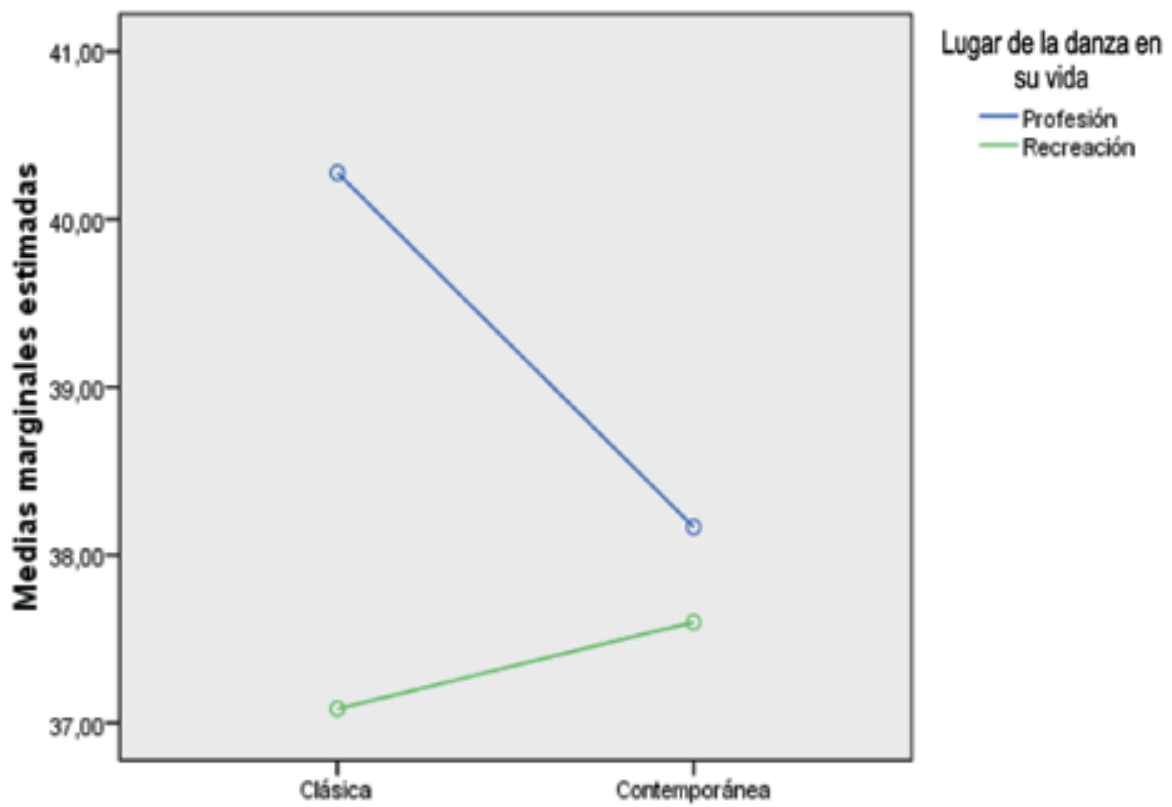

Tipo de danza que desarrolla 


\section{DISCUSIÓN}

El análisis de la hipótesis específica 1 permite establecer que esta no es válida, lo cual quiere decir que no existen diferencias significativas en la ansiedad estado entre bailarines de danza clásica y contemporánea. A pesar de que ambos tipos de danza, como lo mencionan Clabaugh y Morling (2004), se diferencian significativamente en cuanto a técnica y estilo, existen situaciones que los bailarines deben afrontar independientemente del tipo de danza que practiquen. Estas situaciones son, por ejemplo, la rutina de entrenamiento, en la cual, a pesar de ser distinta en ambos casos, el nivel de exigencia busca lograr la perfección en el movimiento; además, en ambos casos los bailarines deben someterse a evaluaciones y concursos en los que deben mostrar su destreza al máximo, independientemente del tipo de danza que practiquen.

Por otro lado, el análisis de la hipótesis específica 2 permite concluir que tampoco es válida, lo cual quiere decir que no existen diferencias significativas en la ansiedad rasgo entre bailarines de danza clásica y contemporánea. Esto demuestra que la ansiedad no es un rasgo de personalidad típico de quienes practican un tipo de danza u otro. Estos resultados se diferencian de los hallados por Clabaugh y Morling (2004), quienes demostraron que los bailarines clásicos y los contemporáneos sí se diferencian en tres rasgos personalidad (autoestima, necesidad personal de estructura y temor a la evaluación negativa). No obstante, Clabaugh y Morling (2004) también encontraron una semejanza en los bailarines de ambos tipos de danza, la cual se vincula con la estima corporal. Tanto las bailarinas de danza clásica como contemporánea no están satisfechas con sus cuerpos y dicha preocupación tiene una importante relación con la ansiedad.

En cuanto a la hipótesis específica 3 , como se puede observar en la tabla 6 , se demuestra que no existen diferencias significativas en la ansiedad estado entre bailarines amateur y profesionales. Esto puede deberse, como ya se mencionó, a que en ambas condiciones (amateur y profesionales) los bailarines buscan alcanzar la plenitud de su potencial y la expresión plena de su talento. Taylor y Taylor (2008) afirman que tanto los bailarines profesionales como los amateur persiguen el grado más elevado de satisfacción personal y artística al bailar; por ello, en ambos casos se enfrentan a situaciones en las cuales deben desarrollar adecuadamente la motivación, la confianza en sí mismos, la intensidad y la concentración.

El análisis de la hipótesis específica 4 evidencia que no existen diferencias significativas en la ansiedad rasgo entre bailarines profesionales y amateur. Recuérdese que, según lo planteado por Spielberger y Díaz-Guerrero (1970), la ansiedad rasgo hace referen- 
cia a las diferencias individuales, relativamente estables, en la propensión a la ansiedad. Es decir que, al parecer, la ansiedad como rasgo de personalidad se manifiesta en niveles similares tanto en bailarines profesionales como amateur. Cabe mencionar que no se han registrado estudios que comprueben o refuten la presencia de diferencias en rasgos de personalidad en bailarines profesionales y amateur. Por tanto, los resultados hallados en este estudio, acerca de la ansiedad estado en bailarines profesionales y amateur, podrían ser un punto de partida para futuras investigaciones.

En referencia a la hipótesis específica 5, el análisis psicométrico evidencia que esta es válida (ver tabla 8). Se encontró que el grupo de los bailarines de danza clásica profesionales poseen mayor ansiedad estado que los bailarines clásicos amateur. Esto contrasta con los resultados obtenidos en los bailarines contemporáneos, puesto que, en este caso, son los contemporáneos amateur quienes presentan más ansiedad estado que los contemporáneos profesionales. Entonces, siguiendo la definición de ansiedad estado propuesta por Spielberger y Díaz-Guerrero (1970), estos resultados estarían indicando que, en la danza clásica, los profesionales se están enfrentando a situaciones que, al parecer, les están ocasionando más ansiedad. En el caso de los contemporáneos, son los amateur lo que están enfrentando situaciones que les están provocando mayor ansiedad.

Todo lo expresado en el párrafo anterior concuerda con lo mencionado en el estudio de Adam, Brassington, Steiner y Matheson (2004), quienes afirman que las bailarinas clásicas profesionales se encuentran expuestas de forma constante a cargas considerables de ansiedad. Estas cargas provienen de las altas expectativas de excelencia artística, la presión de mantener un peso bajo, el horario de entrenamiento exhaustivo (el cual es menor que el de los bailarines clásicos amateur), la competencia feroz y la falta de seguridad en la permanencia en el trabajo.

En cuanto a los bailarines contemporáneos, se encontró que los amateur presentan mayor ansiedad estado que los profesionales. Estos resultados concuerdan con lo planteado en el estudio de Clabaugh y Morling (2004), quienes afirman que la danza contemporánea libera al bailarín de las limitaciones y formalidades de la danza clásica. Además, los autores encontraron que uno de los rasgos que diferencia a los bailarines contemporáneos de los clásicos es que los primeros carecen de la necesidad de cierta estructura en comparación con los segundos. Los autores sugieren que este resultado es el esperado porque en la danza contemporánea se busca que los bailarines improvisen y tengan una interpretación personal de los movimientos, por lo cual este tipo de danza 
atraería a bailarines que posean una alta tolerancia a la ambigüedad.

En relación con la hipótesis específica 6, el análisis estadístico evidencia que no existen diferencias significativas en la ansiedad rasgo entre bailarines de danza clásica profesionales y bailarines de danza clásica amateur, y entre bailarines de danza contemporánea amateur y de danza contemporánea profesionales (ver figura 2). Hasta el momento, no se han reportado estudios que afirmen que existen diferencias significativas en la ansiedad como rasgo de la personalidad de los bailarines pertenecientes a uno u otro grupo de danza.

Entonces, con base en la discusión de las hipótesis específicas 5 y 6 , se puede decir que los bailarines presentan ansiedad, no porque sea un rasgo de personalidad que aparecerá independientemente de la actividad que realicen, sino porque existen situaciones específicas en la danza a las que los bailarines se enfrentan y que les generan ansiedad. Esto último podría estar interfiriendo en su rendimiento óptimo.

\section{Conclusiones}

1. No existen diferencias significativas en la ansiedad estado entre bailarines de danza clásica y danza contemporánea. En otras palabras, la presencia de la ansiedad estado no difiere entre los grupos clásico y contemporáneo.

2. No existen diferencias significativas en la ansiedad rasgo entre bailarines de danza clásica y danza contemporánea. Esto significa que la presencia de la ansiedad como rasgo de personalidad es igual en ambos grupos.

3. No existen diferencias significativas en la ansiedad estado entre bailarines amateur y profesionales, es decir que, al comparar la ansiedad estado de los bailarines profesionales con los bailarines amateur, los resultados son similares.

4. No se han hallado diferencias significativas en la ansiedad rasgo entre bailarines amateur y profesionales, esto es, al comparar la ansiedad como rasgo de personalidad en los dos grupos de bailarines, según su condición como bailarín, se encontraron resultados similares.

5. Existen diferencias significativas en la ansiedad estado entre bailarines de danza clásica profesionales y bailarines de danza clásica amateur, y entre bailarines de danza contemporánea amateur y danza contemporánea profesionales. En otras palabras, los bailarines clásicos profesionales presentan mayor ansiedad estado que los bailarines clásicos amateur. Además, se puede afirmar que los bailarines contemporáneos amateur evidencian mayor ansiedad estado que los contemporáneos profesionales.

6. No se encontró diferencias significativas en la ansiedad rasgo entre bailarines de danza clásica profesionales y bailarines de danza clásica amateur, y entre bailarines de dan- 
za contemporánea amateur y danza contemporánea profesionales. Es decir que, al comparar la ansiedad rasgo en bailarines clásicos profesionales y amateur, se hallaron resultados similares. Asimismo, en el caso de los bailarines contemporáneos profesionales y amateur, la ansiedad como rasgo de personalidad no difiere en uno u otro.

\section{RefERENCIAS}

Adam, M., Brassington, G., Steiner, H., \& Matheson, G. (2004). Factores psicológicos asociados con heridas que limitan el rendimiento en bailarinas profesionales. Journal of Dance Medicine \& Science, 8(2), 43-46. Recuperado el 16 de marzo de 2010 de SPORTDiscus.

American Psychological Association (2010). Manual de Publicaciones de la American Psychological Association. (3. ${ }^{\text {a }}$ ed., Trad.: M. Guerra \& S. Peña Alfaro). México, D. F.: Manual Moderno.

Bárcena, P., Vellido, G., \& Zavala, J. (1994). El hombre y la danza. México, D. F.: Patria. Recuperado el 5 de julio de 2008 de http://www.danzar. com/mp/content/view/20/34/

Castañer, M. (2000). Expresión corporal y danza. Barcelona: INDE Publicaciones.

Clabaugh, A., \& Morling, B. (2004). Precisión de los estereotipos de bailarinas ballet y danza moderna. Journal of Social Psychology, 144(1), 31-48. Recuperado de SPORTDiscus.

Courbebaisse, J. (1974). Gramática de la danza clásica. Buenos Aires: Hachete.

Derka, J., \& Lehotsky, J. (2007). Classical ballet - not only for professional dancers. Gymnica, 37, 40. Recuperado el 8 de abril de 2010 de EBSCO.

Edelmann, R. (1992). Anxiety. Theory, Research and Intervention in Clinical and Health Psychology. West Sussex: Library of Congress.

García, F. (1997). El papel de las emociones en el deporte. Lima: Universidad San Martín de Porres.

Hernández, R., Fernández Collado, C., \& Baptista, L. (2010). Metodología de la investigación. (5. ${ }^{\mathrm{a}}$ ed.). México, D. F.: McGraw-Hill.

Jeffri, J., \& Throsby, D. (2006). Life after Dance: Career Transition of Professional Dancers. International Journal of Arts Management, 8, 5463. Recuperado el 17 de octubre de 2010 de ProQuest.

Kerlinger, F., \& Lee, H. (2001). Investigación del comportamiento. México, D. F.: McGraw-Hill.

Lizárraga, G. (1999). Puesta a punto psicodeportivo: estrategias psicológicas aplicadas a la alta competición. Revista de la Facultad de Psicología 
de la Universidad Nacional Mayor de San Marcos, 4, 23-26.

Nunnally, J., \& Bernstein, I. (1995). Teoría psicométrica. México, D. F.: McGraw-Hill.

Paparizos, A., Tripp, D., Sullivan, M., \& Rubenstein, M. (2005). Catastrofismo y percepción del dolor en bailarinas de ballet recreacionales. Journal of Sport Behavior, 28(1), 35. Recuperado el 16 de marzo de 2010 de SPORTDiscus.

Price, B., \& Pettijohn II, T. (2006). El efecto del atuendo de ballet en el cuerpo y las autopercepciones de las bailarinas. Social Behavior \& Personality: An International Journal, 34(8), 991-998. Recuperado el 31 de marzo de 2010 de SPORTDiscus.

Salazar, A. (1955). La danza y el ballet. México, D. F.: Fondo de Cultura Económica.

Siegel, S. (1991). Estadística no paramétrica: aplicada a las cien- cias de la conducta. México, D. F.: Trillas.

Siegel, S., \& Castellan, J. (1995). Estadística no paramétrica. México, D. F.: Trillas.

Sierra J., Ortega, V., \& Zubeidat, I. (2003). Ansiedad, angustia y estrés: tres conceptos a diferenciar. Revista Mal-estar e Subjetividade, 3(001), 10-59. Fortaleza: Universidad de Fortaleza. Recuperado el 10 de octubre de 2010 de e-libro.

Spielberger, Ch., \& Díaz-Guerrero, R. (1970). Inventario de Ansiedad Rasgo-Estado (IDARE). Florida: University of South Florida.

Taylor, C., \& Taylor, J. (2008). Psicología de la danza. Madrid: Artes Gráficas.

Vallejo, J., \& Gastó, C. (2000). Trastornos afectivos: ansiedad y depresión. Barcelona: MASSON.

Weinberg, R., \& Gould, D. (1996). Fundamentos de psicología del deporte y el ejercicio físico. Barcelona: Ariel. 